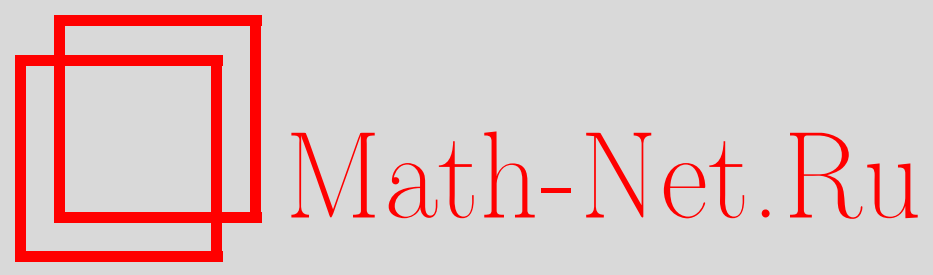

А. П. Колесников, Алгебраические сплайны в локально выпуклых пространствах, Матем. заметки, 2005, том 77, выпуск $3,339-353$

DOI: https://doi.org/10.4213/mzm2497

Использование Общероссийского математического портала Math-Net.Ru подразумевает, что вы прочитали и согласны с пользовательским соглашением http://www . mathnet.ru/rus/agreement

Параметры загрузки:

IP : 54.210 .77 .194

26 апреля 2023 г., 18:19:32

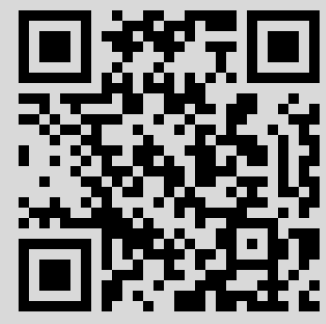




\section{АЛГЕБРАИЧЕСКИЕ СПЛАЙНЫ \\ В ЛОКАЛЬНО ВЫПУКЛЫХ ПРОСТРАНСТВАХ}

\section{А. П. Колесников}

В векторном пространстве непрерьвньх функций найдено вариационное решение конечной системы линейных функциональных уравнений. Выяснено, какой локально вьпуклой топологией нужно наделить векторное пространство и какими должны быть свойства оценочного функционала, чтобы получить искомое решение в форме разложения по базису, двойственному для семейства функционалов системы. Его базисные элементы вычислены точно и названы базисными алгебраическими сплайнами, а построенная по ним линейная оболочка - пространством алгебраических сплайнов в соответствующем локально выпуклом пространстве.

Библиография: 7 названий.

1. Введение. Значительное число задач численного анализа (интерполяции, нахождения слабых решений операторных уравнений в методе Галеркина и т.д.) приводится к конечной системе линейных функциональных уравнений вида $f_{i}(x)=r_{i}, r=\left\{r_{i}\right\}$, $i=1, \ldots, n$ (далее просто системе), где считаются заданньми конечное семейство линейных линейно независимых функционалов $\left\{f_{i}\right\}$ на $\mathbf{E}$ и набор чисел $\left\{r_{i}\right\} ; \mathbf{E}$-векторное пространство, $x \in \mathbf{E}$ - искомьй элемент. Требуется решить следующие вопросы:

1) определить условия (в частности, топологию в $\mathbf{E})$, при которых система разрешима;

2) установить подмножество в $\mathbf{E}$, на котором система имеет единственное решение;

3) построить метод ее решения.

Наибольшее распространение в численном анализе получил способ нахождения решений $x \in \mathbf{E}$ системы с помощью заданных конечномерных апшроксимирующих пространств $\mathbf{E}_{n} \subset \mathbf{E}$. Эта схема решения носит название проекционного метода. Мы рассматриваем способ, при котором базис аппроксимирующего пространства $\mathbf{E}_{n} \subset \mathbf{E}$ не выбирается, а вычисляется исходя из условий задачи и требований к дифференциально-аналитическим свойствам решения. Базис пространства $\mathbf{E}_{n}$ образует семейство функций, дуальное для заданного семейства функционалов $\left\{f_{i}\right\}$. Он определяется как точные вариационные решения системы, которые строятся путем минимизации некоторого оценочного функционала с ограничениями в виде равенств, задаваемых уравнениями системы. За решениями этой вариационной задачи сохраняется термин "алгебраический сплайн" , поскольку таково же вариационное определение классического сплайна в гильбертовых пространствах.

Работа выполнена при поддержке фонда по фундаментальным исследованиям в области авиационной и ракетно-космической техники, грант № 96-17-1. 
2. Порождение векторных пространств. Изучим предварительно алгебраический аспект проблемы порождения локально выпуклых пространств как векторных [1], [2]. Пусть задан изоморфизм $A: \mathbf{E} \rightarrow \mathbf{F}$ векторных пространств $\mathbf{E}$ и $\mathbf{F}$, причем базис пространства $\mathbf{F}$ известен. Существует обратное отображение $A^{-1}=G: \mathbf{F} \rightarrow \mathbf{E}$, и тогда $G\left(\sum \lambda_{\alpha} f_{\alpha}\right)=\sum \lambda_{\alpha} e_{\alpha}, \alpha \in \mathbf{A}$, где $\mathbf{A}-$ произвольное множество индексов и $\left(f_{\alpha}\right)$ - заданный базис в пространстве $\mathbf{F}$. Семейство $\left(e_{\alpha}\right), \alpha \in \mathbf{A}$, определенное равенствами $e_{\alpha}=G\left(f_{\alpha}\right)$, образует базис в пространстве $\mathbf{E}$. В такой постановке задача построения базисов сводится к нахождению изоморфизмов пространств $\mathbf{E}$ и $\mathbf{F}$, при этом пространство $\mathbf{F}$ будем назьвать калибровочным для Е. Для ее решения используем теорию двойственности в векторных пространствах.

Сопряженное или двойственное по отношению к $A$ линейное отображение $A^{*}$ определяется равенством $\left(A^{*} f\right)(x)=f(A x), x \in \mathbf{E}$ и $f \in \mathbf{F}^{*}$, и характеризуется следующими свойствами эквивалентности [1]:

1) $A^{*}$ есть изоморфизм из $\mathbf{F}^{*}$ в $\mathbf{E}^{*} \Longleftrightarrow A$ отображает $\mathbf{E}$ на $\mathbf{F}$;

2) $A^{*}$ отображает $\mathbf{F}^{*}$ на $\mathbf{E}^{*} \Longleftrightarrow A$ есть изоморфизм из $\mathbf{E}$ в $\mathbf{F}$;

3) $A^{*}$ есть изоморфизм $\Longleftrightarrow A$ есть изоморфизм.

Пусть $\mathbf{E}$ - векторное пространство непрерывных числовых функций, определенных на произвольном множестве $\mathbf{T}, x(t) \in \mathbf{E}, t \in \mathbf{T}$ и на $\mathbf{E}$ задан функционал $\delta_{t}$ (точка $t$ фиксирована), действие которого на элемент $x$ определяется так: $\left\langle x, \delta_{t}\right\rangle=x(t)$. Очевидно, он линейньй и тогда $\delta_{t} \in \mathbf{E}^{*}$.

А) Для заданного изоморфизма $A: \mathbf{E} \rightarrow \mathbf{F}$ пространств $\mathbf{E}$ и $\mathbf{F}$ построим обратное для него отображение, используя определение сопряженного оператора $A^{*}: \mathbf{F}^{*} \rightarrow \mathbf{E}^{*}$, осуществляющего изоморфизм пространств $\mathbf{F}^{*}$ и $\mathbf{E}^{*}$. В силу предположения $\delta_{t} \in \mathbf{E}^{*}$ оператор $A^{*}$ характеризуется свойством: для всякого $t \in \mathbf{T}$ в $\mathbf{F}^{*}$ существует элемент $G_{t}$ такой, что вьполнено условие $A^{*} G_{t}=\delta_{t}, G_{t} \in \mathbf{F}^{*}$, т.е. это уравнение разрешимо. Числовая функция $G_{t}(s) \equiv G(t, s): \mathbf{T} \times \mathbf{T} \rightarrow \mathbb{R}-$ фундаментальное решение (функция Грина) оператора $A^{*}$.

Напишем цепочку очевидных равенств: $x(t)=\delta_{t}(x)=\left(A^{*} G_{t}\right)(x)=\left(G_{t}\right)(A x)=$ $G_{t}(f), f \in \mathbf{F}, G_{t} \in \mathbf{F}^{*}$. Равенство $x(t)=G_{t}(f), f \in \mathbf{F}, G_{t} \in \mathbf{F}^{*}$, устанавливает линейное отображение пространства $\mathbf{F}$ на $\mathbf{E}$ и, следовательно, реализует обратное для $A$ отображение $G$. Множество $S=\left\{G_{t}(f)\right\}$, которое образуется, когда линейная форма $f$ пробегает все пространство $\mathbf{F}$, совпадает с $\mathbf{E}$. Таким образом, пространство $\mathbf{F}$ порождает пространство $\mathbf{E}$ и если в нем известен базис $\left(f_{\alpha}\right), \alpha \in \mathbf{A}$, то соответствующий базис в пространстве $\mathbf{E}$ строится по формуле $e_{\alpha}(t)=G_{t}\left(f_{\alpha}\right), \alpha \in \mathbf{A}$.

В) Пусть $A: \mathbf{E} \rightarrow \mathbf{F}$ действует из $\mathbf{E}$ в $\mathbf{F}$, а оператор $A^{*}: \mathbf{F}^{*} \rightarrow \mathbf{E}^{*}$ осуществляет изоморфизм пространства $\mathbf{F}^{*}{ }_{\boldsymbol{B}} \mathbf{E}^{*}$. Тогда $A$ есть отображение на. Если $\delta_{t} \in A^{*}\left(\mathbf{F}^{*}\right)$, то для всякого $t \in \mathbf{T}$ в $\mathbf{F}^{*}$ существует решение уравнения $A^{*} G_{t}=\delta_{t}$ и оказываются справедливьми соотношения $x(t)=\delta_{t}(x)=\left(A^{*} G_{t}\right)(x)=\left(G_{t}\right)(A x)=G_{t}(f), f \in \mathbf{F}$, $G_{t} \in \mathbf{F}^{*}$. Равенство $x(t)=G_{t}(f), f \in \mathbf{F}, G_{t} \in \mathbf{F}^{*}$, реализует обратное отображение $G$ пространства $\mathbf{F}$ на $\mathbf{E}$, т.е. $A: \mathbf{E} \rightarrow \mathbf{F}$ - изоморфизм пространств $\mathbf{E}$ и $\mathbf{F}$. Таково же отображение $A^{*}: \mathbf{F}^{*} \rightarrow \mathbf{E}^{*}$, т.е. $A^{*}\left(\mathbf{F}^{*}\right)=\mathbf{E}^{*}$. В результате мы имеем рассмотренньй случай А).

С) Пусть установлен изоморфизм $A$ пространств $\mathbf{E}$ и $\mathbf{E}^{*}$. Тогда оператор $A^{*}: \mathbf{E}^{* *} \rightarrow$ $\mathbf{E}^{*}$ осуществляет изоморфизм пространств $\mathbf{E}^{* *}$ и $\mathbf{E}^{*}$. В силу условия $\delta_{t} \in \mathbf{E}^{*}$ в $\mathbf{E}^{* *}$ существует элемент $G_{t}$, являюшийся решением уравнения $A^{*} G_{t}=\delta_{t}, G_{t} \in \mathbf{E}^{* *}$, и тогда $x(t)=\delta_{t}(x)=\left(A^{*} G_{t}\right)(x)=\left(G_{t}\right)(A x)=G_{t}(f), f \in \mathbf{E}^{*}, G_{t} \in \mathbf{E}^{* *}$. Равенство 
$x(t)=G_{t}(f), f \in \mathbf{E}^{*}, G_{t} \in \mathbf{E}^{* *}$, определяет обратное отображение $G: \mathbf{E}^{*} \rightarrow \mathbf{E}$. Если при этом окажется, что $G_{t} \in \mathbf{E} \subset \mathbf{E}^{* *}$, то рассматривая его на подпространстве $\mathbf{E}$ в $\mathbf{E}^{* *}$, приходим к равенству

$$
x(t)=f\left(G_{t}\right), \quad f \in \mathbf{E}^{*}, G_{t} \in \mathbf{E} .
$$

Очевидно, что операторы, действуюшие в векторных пространствах, требуется наделить свойством непрерывности, вводя топологии в $\mathbf{E}$ и $\mathbf{E}^{*}$.

Приведенная схема определения порождающих множеств позволяет по заданному линейно независимому семейству $\left(f_{i}\right) \subset \mathbf{E}^{*}, i=1, \ldots, n$, построить двойственный для него алгебраический базис в $\mathbf{E}$. Линейное отображение $G: \mathbf{E}^{*} \rightarrow \mathbf{E}$, определенное равенством (1), переводит семейство $\left(f_{i}\right)$ функционалов, ограниченных на $\mathbf{E}$, в линейно независимое семейство $\left(x_{i}\right): x_{i}=f_{i}\left(G_{t}\right), f_{i} \in \mathbf{E}^{*}, G_{t} \in \mathbf{E}, x_{i} \in \mathbf{E}, i=1, \ldots, n$, образующее алгебраический базис в $\mathbf{E}$ :

$$
f=\sum_{i=1}^{n} \lambda_{i} f_{i} \Longrightarrow x(t)=\sum_{i=1}^{n} \lambda_{i} x_{i}
$$

Легко убедиться, что существует невырожденное линейное преобразование $\left(x_{i}\right) \rightarrow\left(e_{i}\right)$ такое, что $\left(e_{i}\right)$ - семейство в $\mathbf{E}$, дуальное для $\left(f_{i}\right)$.

3. Порождение локально выпуклых пространств. Алгебраическими сплайнами в векторном пространстве $\mathbf{E}$ назовем вариационные решения конечных систем линейных функциональных уравнений, определенных на $\mathbf{E}$. Такие решения будем получать как точные в пространствах с локально вьпуклой топологией для некоторых вариационных задач. Выбор в векторных пространствах локально вьпуклой топологии позволяет использовать теорию двойственности для построения в них базисов. В пространствах Соболева эта задача решена в [3].

Пусть $\mathbf{E}$ в общем случае неотделимое локально выпуклое пространство, $\mathbf{E}^{\prime}$ - сопряженное ему пространство, сильная топология которого определена как отделимая. Элементами пространства $\mathbf{E}$ считаем числовые функции $x: \Omega \rightarrow \mathbb{R}$, где $\Omega$ - ограниченная область в $\mathbb{R}^{m}, \partial \Omega$ - гранища области $\Omega$. Через $K$ обозначим компактное множество $\Omega+\partial \Omega$ в $\mathbb{R}^{m}$. Алгебраические базисы в $\mathbf{E}$ будем находить, строя вариационные решения систем линейных функциональньх уравнений

$$
f_{i}(x)=r_{i}, \quad x \in \mathbf{E}, \quad f_{i} \in \mathbf{E}^{\prime}, \quad i=1, \ldots, n,
$$

где $\left(f_{i}\right) \subset \mathbf{E}^{\prime}$ - семейство линейно независимых функционалов из топологически сопряженного пространства, $\left(r_{i}\right) \subset \mathbb{R}^{n}$ - заданный вектор. Векторы $\left(f_{i}\right),\left(r_{i}\right), i=1, \ldots, n$, условимся обозначать соответственно через $\mathbf{f}$ и $\mathbf{r}$. Систему (2) удобно записать в операторной форме:

$$
\mathbf{f}(x)=\mathbf{r}, \quad \mathbf{f}: \mathbf{E} \rightarrow \mathbb{R}^{n} .
$$

Множество решений системы (3) $D=D(\mathbf{f}, \mathbf{r})=\{x \in \mathbf{E}: \mathbf{f}(x)=\mathbf{r}\}$ характеризуется

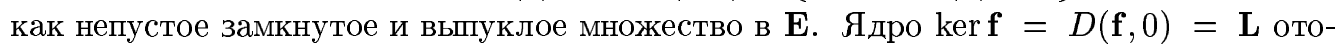
бражения $\mathbf{f}$ есть линейное подпространство, замкнутое в $\mathbf{E}$. Поскольку $D$ - аффинное подпространство в $\mathbf{E}$, существует элемент $x_{0} \in D \backslash \mathbf{L}$ такой, что произвольный элемент $x \in D$ имеет представление $x=x_{0}+h$, где $h \in \mathbf{L}, x_{0}$ - частное решение системы (3). 
Решение системы (3) естественно искать в классе непрерьвных функций. Поскольку она единственным образом не разрешима, к искомому решению предъявим некоторые дополнительные требования, а именно, из множества ее решений выберем те, на которых некоторый оценочньй функционал $f$ принимает минимальное значение. Таким образом, вариационная постановка задачи о решении системы (3) включает минимизируемую функцию $f(x)$ и равенства (3) как линейные ограничения:

$$
\begin{gathered}
f(x) \rightarrow \inf , \quad x \in \mathbf{E}, \\
\mathbf{f}(x)=\mathbf{r} .
\end{gathered}
$$

Ограничения (5) назовем уравнениями связей или интерполящионными условиями в задаче (4), (5). Ее удобно записать в следующей компактной форме:

$$
f(x) \rightarrow \inf , \quad x \in D \subset \mathbf{E},
$$

где $D$ - непустое множество допустимых элементов.

4. Задача условной минимизации. Покажем, что задачу условной минимизации (6) можно привести к эквивалентной задаче безусловной минимизации функции Лагранжа, в которой множители Лагранжа выбираются так, чтобы удовлетворялись условия (5). Соответствующее утверждение формулирует

ТЕорема 1 (Эйлера). Пусть әлемент $a \in D$ решает задачу (6). Тогда найдутся такие не равные одновременно нулю множители Лагранжа $\left(\lambda_{j}\right)$, что точка а будет стачионарной для функиионала

$$
F(x)=f(x)-\sum_{j=1}^{n} \lambda_{j} f_{j}(x), \quad x \in \mathbf{E}, \quad f_{j} \in \mathbf{E}^{\prime}
$$

При этом $\lambda_{j}$ будут находиться из условий (5).

Данная теорема, содержание которой восходит к изопериметрической задаче Эйлера, формулирует правило множителей Лагранжа. Доказательство ее можно найти в стандартных курсах функционального анализа. Обычно оно дается для общего случая нелинейных функционалов $\left(f_{j}\right)$ и в нашем случае мало полезно. Мы приведем простое доказательство этой теоремы, учитывающее, что функционалы $f_{j}$ линейны.

ДоказАтЕльство. Выберем линейно независимые элементы $h_{0}, \ldots, h_{n}, h_{k} \in \mathbf{E}$, $k=0, \ldots, n$, и соответствующие вещественные переменные $\alpha_{0}, \ldots, \alpha_{n}$. Вариацию $a+h$ точки $a$ возьмем в виде $x=a+h=a+\sum_{k=0}^{n} \alpha_{k} h_{k}$, где $a-$ точка из $D$, решаюшая задачу (6), и будем считать, что $h_{1}, \ldots, h_{n}$ - элементы, дуальные для $\left(f_{i}\right)$, а коэффициенты $\left(\alpha_{k}\right), k=1, \ldots, n$, выберем так, чтобы вариация $a+h$ в точке $a \in D$ не выводила $x$ за пределы множества $D$, т.е. $a+h \in D$. При этом $\alpha_{0}$ и $h_{0} \in \mathbf{E}$ остаются произвольными. Условие $a+h \in D$ означает, что

$$
f_{i}\left(a+\sum_{j=0}^{n} \alpha_{j} h_{j}\right)=r_{i}, \quad i=1, \ldots, n
$$


Поскольку элемент $a \in D$ удовлетворяет условиям (5), имеем систему линейных функциональных уравнений $\sum_{j=1}^{n} f_{i}\left(h_{j}\right) \alpha_{j}=-\alpha_{0} f_{i}\left(h_{0}\right), i=1, \ldots, n$, относительно $\alpha_{j} . \mathrm{B}$ силу ортогональности векторов $\left(f_{i}\right) \subset \mathbf{E}^{\prime}$ и $\left(h_{i}\right) \in \mathbf{E}, i=1, \ldots, n$, решение системы $(8)$ получим в виде $\alpha_{j}=-\alpha_{0} f_{j}\left(h_{0}\right), j=1, \ldots, n$. Подставляя вычисленные коэффициенты в уравнения (8), превратим их в тождества $f_{i}\left(a+\alpha_{0} h_{0}+\alpha_{0} \sum_{j=1}^{n} \beta_{j} h_{j}\right) \equiv r_{i}$, $i=1, \ldots, n, \beta_{j}=-f_{j}\left(h_{0}\right)$, удовлетворяющиеся при любом значении $\alpha_{0}$. Заметим, что при $\alpha_{0}=0$ справедливо равенство $x(0)=a$, а поскольку $f(x)$ имеет минимум в точке $a$, утверждается, что

$$
\left.\frac{d}{d \alpha_{0}} f\left(x\left(\alpha_{0}\right)\right)\right|_{\alpha_{0}=0}=\delta f\left(a, h_{0}\right)+\sum_{j=1}^{n} \delta f\left(a, h_{j}\right) \beta_{j}=\delta f\left(a, h_{0}\right)-\sum_{j=1}^{n} \lambda_{j} f_{j}\left(h_{0}\right)=0
$$

где положено $\lambda_{j}=\delta f\left(a, h_{j}\right)$. Если обозначить $F(x)=f(x)-\sum_{j=1}^{n} \lambda_{j} f_{j}(x)$, то легко увидеть, что это утверждение есть условие стационарности функционала $F(x)$ :

$$
\delta F(x, h)=\delta f(x, h)-\sum_{j=1}^{n} \lambda_{j} f_{j}(h)=0, \quad x \in \mathbf{E}, \quad h \in \mathbf{E},
$$

в точке $a$, что и доказьвает теорему.

5. Решение задачи условной минимизации. Известно [4], что пространство $\mathbf{E}$ разлагается в прямую топологическую сумму $\mathbf{E}=\mathbf{E}_{n} \oplus \operatorname{ker} \mathbf{f}$, где $\mathbf{E}_{n}$ - векторное подпространство, порожденное элементами $\left(e_{i}\right)$, дуальными семейству $\left(f_{i}\right)$. Это утверждение по сути лишь отмечает факт существования в $\mathbf{E}$ базисных элементов $\left(e_{i}\right)$ таких, что каждый вектор $x \in \mathbf{E}$ может быть представлен в виде разложения $x=\sum_{i=1}^{n} f_{i}(x) e_{i}+\varphi$, $\varphi \in \operatorname{ker} \mathbf{f}$, однако оно не содержит указаний на способ, с помощью которого этот базис мог бы быть найден.

Дуальньй для $\left(f_{i}\right)$ алгебраический базис в $\mathbf{E}$ вьгислим, построив точное решение задачи (6). Ее постановка является слишком общей, чтобы можно было рассчитывать на получение решений в аналитической форме. Мы не будем предполагать, что пространство $\mathbf{E}$ отделимо, поэтому если сушествует решение задачи (6), то оно не единственно. В E образуем ассоциированное факторпространство $\mathscr{E}=\mathbf{E} /\{\overline{0}\}$, фактортопология которого отделима. Выясним некоторые наиболее общие требования к топологии пространства $\mathbf{E}$ и функционалу $f$, чтобы получить разрешимую вариационную задачу.

(1) Предположим, что в $\mathscr{E}$ можно выделить всюду плотное подмножество элементов $\mathscr{X}$ такое, что функционал $f$ дважды дифференцируем по Гато в каждой точке $x \in \mathscr{X}$, т.е. на множестве $\mathscr{X}$ определено слабо непрерьвное симметрическое отображение $\mathbf{P}: \mathscr{X} \rightarrow$ $\mathbf{P}(\mathscr{X})=X^{\prime}$ (оператор Эйлера для функционала $f$ ), являющееся второй производной функционала $f(x))$. Заметим, что в вариационном исчислении непрерывность оператора $\mathbf{P}$ всегда проявляется в слабой форме, поэтому говоря о непрерьвности $\mathbf{P}$, мы будем иметь это ввиду, но специально не всегда оговаривать. Обычно $\mathscr{X}$ - это подмножество в $\mathscr{E}$, на элементы которого накладываются некоторые дополнительные ограничения, не содержащиеся в исходной постановке вариационной задачи, например, на их поведение в точках границы. Будем принимать во внимание лишь такие функционалы $f(x)$, для которых $\mathbf{P}$ не зависит от $x$. Пусть также в силу выбора $f(x)$ оператор $\mathbf{P}$ положительный. Это означает, что квадратичная форма $\langle\mathbf{x}, \mathbf{P x}\rangle, \mathbf{x} \in \mathscr{X}$, положительно определена:

$$
\langle\mathbf{x}, \mathbf{P x}\rangle \geqslant 0 \text { и }\langle\mathbf{x}, \mathbf{P x}\rangle=0 \Longrightarrow \mathbf{x}=0 .
$$


Поскольку $\operatorname{ker} \mathbf{P}=0$, то $\mathbf{P}$ - алгебраический изоморфизм пространств $\mathscr{X}$ и $X^{\prime}$. При некоторых ограничениях на топологии пространств $\mathbf{E}$ и $X^{\prime}$, например, когда $\mathbf{E}-$ совершенно полное пространство, а $X^{\prime}$ - бочечно, что мы и будем считать в дальнейшем, это слабый изоморфизм пространств $\mathscr{X}$ и $X^{\prime}$ как топологических (теорема Банаха о гомоморфизме). Обратным для оператора $\mathbf{P}$ является слабо непрерьвное отображение $\mathbf{G}=\mathbf{P}^{-1}: X^{\prime} \rightarrow \mathscr{X}$ и решение уравнения $\mathbf{P} \mathbf{x}=f, f \in X^{\prime}$, записывается в этом случае в слабой форме: $\langle\mathbf{G} f, g\rangle=\langle\mathbf{x}, g\rangle, \mathbf{x} \in \mathscr{X}, f \in X^{\prime}, g \in X^{\prime}$ - произвольный элемент. В силу симметричности $\mathbf{P}:\langle\mathbf{y}, \mathbf{P x}\rangle=\langle\mathbf{x}, \mathbf{P y}\rangle, \mathbf{x}, \mathbf{y} \in \mathscr{X}$ оператор $\mathbf{G}$ симметрический: $\langle\mathbf{G} g, f\rangle=\langle\mathbf{G} f, g\rangle, f, g \in X^{\prime}$, а положительность $\mathbf{P}$ влечет аналогичное свойство оператора $\mathbf{G}$.

(2) Предметом нашего рассмотрения будут только векторные пространства непрерьвных функций. Это означает, что мера Дирака $\delta_{t}$ с носителем в точке $t \in K$ определена на элементах $\mathbf{x} \in \mathscr{X}: \delta_{t} \in X^{\prime}$. Тогда существует слабое решение $\mathbf{G}_{t} \in \mathscr{X}$ уравнения $\mathbf{P G}_{t}=\delta_{t}$ относительно точки $t \in K$ и можно положить $\left\langle\mathbf{G} \delta_{t}, f\right\rangle=\left\langle\mathbf{G}_{t}, f\right\rangle, f \in X^{\prime}$. Функцию $\mathbf{G}_{t}$ назьвают фундаментальным решением оператора $\mathbf{P}$. Вследствие симметричности отображения $\mathbf{G}$ функция $\mathbf{G}_{t}$, зависящая от двух аргументов, симметрична относительно их перестановки: $\mathbf{G}_{t}(s) \equiv \mathbf{G}(t, s)=\mathbf{G}(s, t), t, s \in K$, и ее назьвают ядром отображения $\mathbf{G}$. В силу перечисленньх вьше свойств оператора $\mathbf{G}$ справедливы равенства

$$
\left\langle\mathbf{G} \delta_{t}, f\right\rangle=\left\langle\mathbf{G} f, \delta_{t}\right\rangle=\left\langle\mathbf{x}, \delta_{t}\right\rangle=\mathbf{x}(t)=\left\langle\mathbf{G}_{t}, f\right\rangle
$$

которые определяют отображение $\mathbf{G}: X^{\prime} \rightarrow \mathscr{X}$ :

$$
\mathbf{x}(t)=f\left(\mathbf{G}_{t}\right), \quad \mathbf{x} \in \mathscr{X}, \quad f \in X^{\prime}, \mathbf{G}_{t} \in \mathscr{X} \quad \text { для всякого } t \in K .
$$

(3) Пусть $\left(f_{i}\right) \subset X^{\prime}$. Обозначим через $\mathscr{A}$ класс матричных операторов с элементами $A_{i j}=f_{i}\left(\varphi_{j}\right), \varphi_{j}=f_{j}\left(\mathbf{G}_{t}\right)$. Следствием симметричности и положительности оператора $\mathbf{G}$ является симметричность и положительность матриц класса $\mathscr{A}$ :

$$
\sum_{i=1}^{n} \sum_{j=1}^{n} \lambda_{i} \lambda_{j} A_{i j}>0
$$

где $\lambda_{i}, \lambda_{j}$ - произвольные вещественные числа, не все равные нулю, т.е. матрицы класса $\mathscr{A}$ неособенные. Положим $\mathscr{W}=(\mathscr{A})^{-1}, \mathscr{W}$ - класс матричных операторов с матричными элементами $W_{i j}$.

Задачу (6) удобно рассмотреть в форме

$$
f(x) \rightarrow \inf , \quad x \in \mathbf{D}, \quad \mathbf{D}=\mathbf{D}(\mathbf{f}, \mathbf{r})
$$

где $\mathbf{D}(\mathbf{f}, \mathbf{r})=\{\mathbf{x} \in \mathscr{X}: \mathbf{f}(x)=\mathbf{r}\}-$ аффинное подпространство в $\mathscr{X}$. Взяв в формуле (9) элемент $f \in X^{\prime}$ в виде линейной комбинации функций $\left(f_{j}\right) \subset X^{\prime}: f=\sum_{j=1}^{n} \lambda_{j} f_{j}$, получим частное решение $\mathbf{x}_{0}(t)=\sum_{j=1}^{n} \lambda_{j} f_{j}\left(\mathbf{G}_{t}\right)$ задачи $\mathbf{f}(\mathbf{x})=\mathbf{r}$, если коэффициенты $\left(\lambda_{j}\right)$ выбрать так, чтобы эти равенства удовлетворялись. Покажем, что построенное решение совпадает с решением вариационной задачи (10).

Следующая теорема содержит утверждение о существовании решений вариационной задачи (10) и приводит их в точной аналитической форме. 
Teорема 2. В силу условий (1)-(3)

а) частное решение задачи (10) существует и определяется равенством

$$
\mathbf{x}_{0}=\sum_{j=1}^{n} \lambda_{j} \varphi_{j}, \quad x_{0} \in \mathbf{D}
$$

в котором коэффичиенты $\left(\lambda_{j}\right)$ находятся из уравнений связи $\mathbf{f}(x)=\mathbf{r}, n p и-$ водящих $x$ системе линейных алгебраических уравнений

$$
\sum_{i=1}^{n} A_{i j} \lambda_{j}=r_{i}
$$

b) все множество решений задачи (10) определяется равенством

$$
\mathbf{x}=\sum_{j=1}^{n} \lambda_{j} \varphi_{j}+\varphi, \quad \varphi \in \operatorname{ker} \mathbf{f}, \quad \mathbf{x} \in \mathbf{D}
$$

c) для подпространства $X_{n}^{\prime} \subset X^{\prime}$, порожденного линейно независимыми әлементами $\left(f_{i}\right)$, существует дуальное ему подпространство $\mathscr{X}_{n} \subset \mathscr{X}$, базисные әлементы $\left(e_{i}\right), i=1, \ldots, n$, которого удовлетворяют условию $f_{j}\left(e_{i}\right)=$ $\delta_{i j}$ и строятся по правилу $e_{i}=\sum_{j=1}^{n} W_{i j} \varphi_{j}$. Пространство $\mathscr{X}$ разлагается в прямую топологическую сумму $\mathscr{X}=\mathscr{X}_{n} \oplus \operatorname{ker} \mathbf{f}$. Данное разложсение означает, что произвольный әлемент $\mathbf{x} \in \mathscr{X}$ может быть единственным образом представлен суммой $\mathbf{x}=\mathbf{P}_{n} \mathbf{x}+\varphi$, әде $\mathbf{P}_{n}-$ непрерывный проектор $\mathbf{P}_{n}: \mathscr{X} \rightarrow \mathscr{X}_{n}$, определенный формулой $\mathbf{P}_{n} \mathbf{x}=\sum_{j=1}^{n} f_{j}(\mathbf{x}) e_{j}$, a $\varphi \in \operatorname{ker} \mathbf{f}$. Частное решение системы (10) при әтом непосредственно выражсается через вектор правых частей: $\mathbf{x}_{0}=\sum_{j=1}^{n} r_{j} e_{j}$.

ДокАЗАТЕЛЬСтво. В силу условия (1) существует вторая вариационная производная функционала $f(x)$ и его вариация определена так:

$$
\delta f(\mathbf{x}, \mathbf{h})=\langle\mathbf{h}, \mathbf{P x}\rangle \equiv(\mathbf{P x})(\mathbf{h}), \quad \mathbf{x} \in \mathscr{X}, \mathbf{h} \in \mathscr{E} .
$$

Решая задачу условной минимизации (10) методом неопределенных множителей Лагранжа, необходимое условие минимума для функционала (7), определенного на $\mathscr{E}$, согласно теореме 1 получаем в виде

$$
\delta F(\mathbf{x}, \mathbf{h})=(\mathbf{P x})(\mathbf{h})-\sum_{j=1}^{n} \lambda_{j} f_{j}(\mathbf{h})=0, \quad \mathbf{x} \in \mathscr{X}, \quad \mathbf{h} \in \mathscr{E} .
$$

В силу произвола в выборе $h$ справедливо равенство

$$
\mathbf{P x}-\sum_{j=1}^{n} \lambda_{j} f_{j}=0, \quad \mathbf{x} \in \mathscr{X} .
$$


Уравнение (12) назьвают уравнением Эйлера-Лагранжа задачи (10). Оно означает, что класс $\mathscr{X}$ образуют точки, стационарные для функции Лагранжа $F(\mathbf{x})$. Необходимое условие минимума в задаче (10) на условньй экстремум при некотором выборе множителей Лагранжа совпадает с необходимым условием безусловного минимума по $\mathbf{x}$ функции Лагранжа $F(\mathbf{x})$, которое в силу положительности оператора $\mathbf{P}$ оказьвается и достаточньм.

Преобразуем равенство (11), используя формулу $\mathbf{h}(t)=\delta_{t}(\mathbf{h}), t \in K, \mathbf{h} \in \mathscr{E}$, справедливую по условию (2). Пусть фундаментальное решение $\mathbf{G}_{t} \in \mathscr{X}$ оператора $\mathbf{P}$ сушествует и обрашает уравнение $\mathbf{P G}_{t}=\delta_{t}$ в тождество. Это позволяет в последнем слагаемом формулы (11) заменить $\mathbf{h}$ на $\mathbf{P} \mathbf{G}_{t}(\mathbf{h})$ :

$$
f_{j}(\mathbf{h})=f_{j}^{t}\left(\delta_{t}(\mathbf{h})\right)=f_{j}^{t}\left(\mathbf{P} \mathbf{G}_{t}\right)(\mathbf{h})=\mathbf{P}\left(f_{j}^{t} \mathbf{G}_{t}\right)(\mathbf{h})
$$

В последнем равенстве мы переставили местами отображения $\mathbf{P}$ и $f_{j}$, действующие на разные переменные (верхний индекс $t$ у функционала $f_{j}$ означает, что он действует на переменную $t)$. Это допустимо, поскольку функционалы $\left(f_{j}\right)$ и оператор $\mathbf{P}$ непрерывны на $\mathbf{G}_{t}$. Так как $\mathbf{h}$ - произвольньй элемент из $\mathscr{E}$, а оператор $\mathbf{P}$ линейньй, равенство (11) в силу (13) преобразуется к виду $\mathbf{P}\left(\mathbf{x}-\sum_{j=1}^{n} \lambda_{j} f_{j}^{t}\left(\mathbf{G}_{t}\right)\right)=0$. Поскольку $\operatorname{ker} \mathbf{P}=0$, в качестве частного решения задачи (10) получаем класс элементов

$$
\mathbf{x}_{0}=\sum_{j=1}^{n} \lambda_{j} f_{j}^{t}\left(\mathbf{G}_{t}\right)=\sum_{j=1}^{n} \lambda_{j} \varphi_{j}, \quad \mathbf{x}_{0} \in \mathbf{D} .
$$

Произвольное решение из множества $\mathbf{D}$ получим добавлением в правую часть равенства (14) слагаемого из ker f:

$$
\mathbf{x}=\sum_{j=1}^{n} \lambda_{j} \varphi_{j}+\varphi, \quad \varphi=\operatorname{ker} \mathbf{f}, \quad \mathbf{x} \in \mathbf{D} .
$$

Коэффициенты $\left(\lambda_{j}\right)$ в решениях $(14),(15)$ находятся из условий связи $\mathbf{f}(\mathbf{x})=\mathbf{r}$.

Базисные функции $\varphi_{j}(t)$ линейно независимы, поскольку получены в результате невырожденного преобразования $\mathbf{G}: X^{\prime} \rightarrow \mathscr{X}$ линейно независимого семейства $\left(f_{j}\right) \subset X^{\prime}$, и образуют алгебраический базис в $\mathscr{X}$. Используя уравнения связи $\mathbf{f}(\mathbf{x})=\mathbf{r}$, получим систему линейных алгебраических уравнений относительно искомых коэффициентов $\left(\lambda_{j}\right): \mathscr{A} \lambda=\mathbf{r}$ с определенными выше невырожденными в силу условия (3) матрицами $\left(A_{i j}\right)$, которая однозначно разрешима. Легко видеть, что базис $\left(e_{i}\right)$, дуальньй для линейно независимого семейства $\left(f_{j}\right)$, получается из системы $\left(\varphi_{j}\right)$ преобразованием $e_{i}=\sum_{j=1}^{n} W_{i j} \varphi_{j}$ с матрицей $\left(W_{i j}\right)$ класса $\mathscr{W}$. Тогда для любого элемента $\mathbf{x} \in \mathscr{X}$ справедливо разложение

$$
\mathbf{x}=\sum_{j=1}^{n} f_{j}(\mathbf{x}) e_{j}+\varphi, \quad \mathbf{x} \in \mathscr{X}, \quad \varphi \in \operatorname{ker} \mathbf{f}
$$

Линейную оболочку, порожденную элементами $\left(e_{j}\right)$, обозначим через $\mathscr{X}_{n}$. Формулой $\mathbf{x}_{n}=\sum_{j=1}^{n} f_{j}(\mathbf{x}) e_{j}, \mathbf{x} \in \mathscr{X}, \mathbf{x}_{n} \in \mathscr{X}_{n}$, определен оператор проектирования $\mathbf{P}_{n}: \mathscr{X} \rightarrow$ 
$\mathscr{X}_{n}$. Алгебраическим дополнением к $\mathscr{X}_{n}$ в $\mathscr{X}$ является подпространство ker $\mathbf{P}_{n}$. Функционалы семейства $\left(f_{j}\right)$ непрерывны на $\mathscr{X}$, поэтому проектор $\mathbf{P}_{n}$ непрерывен и алгебраическая прямая сумма $\mathscr{X}=\mathscr{X}_{n} \oplus \operatorname{ker} \mathbf{P}_{n}$ будет топологической прямой суммой. Легко видеть, что $\operatorname{ker} \mathbf{P}_{n}=\operatorname{ker} \mathbf{f}$. Для частного решения $\mathbf{x}_{0}$ задачи (10) из (16) имеем $\mathbf{x}_{0}=\sum_{j=1}^{n} r_{j} e_{j}, \mathbf{x}_{0} \in \mathbf{D}$.

Пространство $\mathscr{X}_{n}$ назовем пространством алгебраических сплайнов в $\mathbf{E}$, порожденных семейством $\left(f_{i}\right)$.

ЗАмЕчАнИЕ 1. Используя уравнение Эйлера-Лагранжа (12) и свойство симметричности оператора $\mathbf{P}$ :

$$
\mathbf{x}(t)=\left\langle\mathbf{x}, \delta_{t}\right\rangle=\left\langle\mathbf{x}, \mathbf{P} \mathbf{G}_{t}\right\rangle=\left\langle\mathbf{G}_{t}, \mathbf{P} \mathbf{x}\right\rangle=\sum_{j=1}^{n} \lambda_{j} f_{j}\left(\mathbf{G}_{t}\right), \quad \mathbf{G}_{t} \in \mathscr{X}, \quad \mathbf{x} \in \mathbf{D}
$$

приходим к решению $\mathbf{x}(t)=\sum_{j=1}^{n} \lambda_{j} f_{j}\left(\mathbf{G}_{t}\right)$ задачи $(10)$, совпадаюшему с решением (14) в силу симметричности функции $\mathbf{G}(t, s)$.

ЗАмечаниЕ 2. Решая вариационную задачу (6) на $\mathbf{E}$, уравнение Эйлера-Лагранжа получаем в форме $P\left(x-\sum_{j=1}^{n} \lambda_{j} f_{j}^{t}\left(G_{t}\right)\right)=0$, где $P$ - заданньй на всюду плотном подпространстве $X$ в $\mathbf{E}$ непрерьвньй оператор Эйлера, $x \in X, G_{t} \in X$. Поскольку $\mathbf{E}$ локально выпукло, он может быть непрерывным образом продолжен на все пространство Е. Тем самым будет определен непрерывный оператор $Q$ (расширение оператора $P$ ), действующий из $\mathbf{E}$ в сопряженное пространство $\mathbf{E}^{\prime}$. Может оказаться, что $\operatorname{ker} Q \neq\{0\}$. В пространстве Е образуем факторпространство $\mathscr{Y}=\mathbf{E} / \operatorname{ker} Q$ и определим непрерывное фактор-отображение $\mathbf{P}: \mathscr{Y} \rightarrow \mathbf{E}^{\prime}$ по правилу $\langle h, \mathbf{P x}\rangle=\langle h, Q x\rangle, \mathbf{x} \in \mathscr{Y}, x \in \mathbf{x}, h \in \mathbf{E}$. Если $\delta_{t} \in \mathbf{E}^{\prime}$, то существует фундаментальное решение $\mathbf{G}_{t}$ оператора $\mathbf{P}$ и с помошю равенства

$$
\mathbf{x}_{0}=\sum_{j=1}^{n} \lambda_{j} f_{j}^{t}\left(\mathbf{G}_{t}\right)=\sum_{j=1}^{n} \lambda_{j} \varphi_{j}, \quad \mathbf{x}_{0}, \mathbf{G}_{t} \in \mathscr{Y}
$$

строится класс частных решений задачи (6), причем коэффициенты находятся из уравнений связи $\mathbf{f}(\mathbf{x})=\mathbf{r}$. Произвольное решение задачи (6) получаем добавлением в правую часть равенства (17) слагаемого из $\operatorname{ker} \mathbf{f}$ :

$$
\mathbf{x}=\sum_{j=1}^{n} \lambda_{j} \varphi_{j}+\varphi, \quad \mathbf{x} \in \mathscr{Y}, \varphi \in \operatorname{ker} \mathbf{f}
$$

Рассмотрим ситуацию, когда условие $(2)$ не вьполнено, т.е. $\delta_{t} \notin X^{\prime}$, но фундаментальное решение оператора $P$ сушествует и принадлежит более широкому пространству, чем Е. К этому случаю проведенное рассмотрение не применимо. Опишем некоторьй общий подход к решению таких задач, опирающийся на теорию двойственности в локально вьпуклых пространствах. Пусть $\mathbf{E}$ - пространство Фреше и заданный оператор $A$ переводит элементы из $\mathbf{E}$ в гильбертово пространство $\mathbf{H}, A: \mathbf{E} \rightarrow \mathbf{H}, \delta_{t} \notin \mathbf{H}$. Тогда соответствующий сопряженньй ему оператор $A^{\prime}: \mathbf{H}^{\prime} \rightarrow \mathbf{E}^{\prime}$ определяется равенством $\langle A x, y\rangle=\left\langle x, A^{\prime} y\right\rangle, x \in \mathbf{E}, y \in \mathbf{H}^{\prime}$. Из предположений

1) $\delta_{t} \in A^{\prime}\left(\mathbf{H}^{\prime}\right) \subset \mathbf{E}^{\prime}$

2) $A^{\prime}$ - слабьй изоморфизм пространства $\mathbf{H}^{\prime}$ в $\mathbf{E}^{\prime}$ 
вытекает, что уравнение $A^{\prime} G_{t}=\delta_{t}$ слабо разрешимо (сушествует единственный элемент $G_{t} \in \mathbf{H}^{\prime}$ для всякого $\left.t \in \Omega\right)$, а $A$ есть топологический изоморфизм пространств $\mathbf{E}$ и $\mathbf{H}$ [4]. В результате получаем реализацию непрерывного отображения $G: \mathbf{H} \rightarrow \mathbf{E}$, $G=A^{-1}$ в виде $x=\left\langle h, G_{t}\right\rangle, h \in \mathbf{H}$. Пусть, например, $\mathbf{H}=\mathbf{H}^{\prime}=L^{2}(\Omega)$. Двойственность в $L^{2}(\Omega)$ определяется с помощью интеграла Лебега: $x=\left(G_{t}, h\right)=\int_{\Omega} h G_{t} d \mu$, $h \in \mathbf{H}, G_{t} \in \mathbf{H}, x \in \mathbf{E}$, устанавливающего непрерьвное отображение $G: L^{2}(\Omega) \rightarrow \mathbf{E}$. Если некоторьй элемент $h \in \mathbf{H}$ представлен разложением по линейно независимой системе функций $\left(h_{j}\right) \subset \mathbf{H}: h=\sum_{j=1}^{n} \lambda_{j} h_{j}$, то равенством

$$
x(t)=\sum_{j=1}^{n} \lambda_{j}\left(h_{j}, G_{t}\right), \quad x \in D, \quad G_{t} \in L^{2}(\Omega)
$$

определяется частное решение системы уравнений $\left(h_{i}, x\right)=r_{i}, i=1, \ldots, n$, в $\mathbf{E}$, которая в свою очередь используется для нахождения коэффициентов $\left(\lambda_{j}\right)$ в решении $(19)$.

Для функции $G_{t} \in L^{2}(\Omega)$, сингулярной в точке $s=t$, соответствующее ядро $G(t, s)$, $t, s \in \Omega$, будем называть сингулярным. Тем самым, накладываются определенные ограничения на вид функционалов $\left(f_{j}\right)$ в задаче $(1)$ : они должны быть элементами пространства $L^{2}(\Omega)$. Тогда система уравнений (1) может быть разрешена в $\mathbf{E}$, функции $\varphi_{j}(t)=\left(f_{j}, G_{t}\right)$ непрерьвны и для решения системы $(1)$ возможно представление

$$
x(t)=\sum_{j=1}^{n} \lambda_{j}\left(f_{j}, G_{t}\right), \quad x \in D, \quad G_{t} \in L^{2}(\Omega) .
$$

Пусть интерполящионные условия заданы равенствами (5), а функционал $f(x)$ определяет функциональный класс, которому должно принадлежать приближение $x_{n}=$ $\sum_{i=1}^{n} \lambda_{i} \varphi_{i}, i=1, \ldots, n$. Если $\mathbf{E}$ неотделимо, то решение $x_{n} \in \mathbf{E}$ зависит от выбора элемента $G_{t} \in \mathbf{G}_{t}$. Мы получаем, таким образом, возможность в одном и том же пространстве $\mathbf{E}$ базисные сплайны $\left(e_{i}\right)$ выбирать различньми способами.

6. Примеры. Следующие ниже примеры показывают применение теоремы 2 к теории приближений. Пусть $\mathbf{E}$ - вещественное векторное пространство функций, определенных на $[a, b]$, с полускалярным произведением $(x \mid y)=\int_{a}^{b}(T x)(T y) d t(T-$ линейный дифференциальный оператор, действующий в $\mathbf{E}$ ) и соответствующей полунормой $p(x)=(x \mid y)^{1 / 2}$. Множество функций $\{x \in \mathbf{E}: p(x)=0\}$ образует ядро отображения $p(x)$. Для $T=d^{m} / d t^{m}$ ядром отображения $p(x)$ будут многочлены степени $(m-1)$. Пространство $\mathbf{E}$ как топологическое локально вьпукло. Обозначим $D=d / d t$ и пусть оператор $T$ задан в форме многочлена $T=D^{m}+a_{1} D^{m-1}+\cdots+a_{m-1} D^{1}+a_{m}$ с постоянными коэффициентами. Тогда $P=T^{*} T$, где $T^{*}=(-1)^{m} D^{m}+(-1)^{m-1} a_{1} D^{m-1}+$ $\cdots-a_{m-1} D^{1}+a_{m}$ - оператор, формально сопряженный с $T$. Вычисляя фундаментальные решения уравнения $P G(t)=\delta(t)$, построим некоторые регулярные типы сплайнов. Считаем, что $f=\sum_{i=1}^{n} \lambda_{i} \delta_{i}\left(t-t_{i}\right), t_{i} \in[a, b], t_{1}=a, t_{n}=b$. Функцию $\varphi(t)=G(t)$ будем назьвать производящей для сплайна $\sum_{i=1}^{n} \lambda_{i} G_{i}\left(t-t_{i}\right)$, а точки $\left(t_{i}\right)$ - опорньми для соответствующих базисных сплайнов $\varphi_{i}(t)=G\left(t-t_{i}\right)$. Если они расположены на $[a, b]$ равномерно с шагом $h$ и в построении решения $G(t)$ участвуют финитные функции, то базисная функция $\varphi_{i}(t)$ получается из $G(t)$ преобразованиями сдвига и изменения масштаба: $\varphi_{i}(t)=G\left(\left(t-t_{i}\right) / h\right)$. Базисы $\left(\varphi_{i}(t)\right), i=1, \ldots, n$, назьвают сеточными. 
А) Многочленные сплайны $\left(a_{j}=0, j=1, \ldots, m, P=(-1)^{m} D^{2 m}\right)$.

1) Глобально определенные сплайны (G-сплайны). Частное решение уравнения $P G(t)=\delta(t)$ возьмем в виде

$$
\varphi(t)=g_{2 m-1}(t)=\frac{(-1)^{m}|t|^{2 m-1}}{2(2 m-1) !}, \quad m=1,2, \ldots,
$$

и формулой $\varphi_{i}(t)=g_{2 m-1}\left(t-t_{i}\right)$ определим базисные сплайны. Очевидно, что линейная комбинация $\sum_{i=1}^{n} \lambda_{i} \varphi_{i}\left(t-t_{i}\right)$ является эквивалентной формой представления кусочно-многочленного сплайна степени $(2 m-1)$, поскольку она:

a) на каждом интервале $\left(t_{i-1}, t_{i}\right), i=2, \ldots, n$, суть многочлен степени $(2 m-1)$,

б) принадлежит классу функций $C^{2 m-2}[a, b]$.

2) Финитные сплайны (В-сплайны). Оператор $P$ удобно записать в форме $P=A^{m}$, $A=-d^{2} / d t^{2}$. Воспользовавшись решением краевой задачи

$$
A G_{1}(t)=\delta(t), \quad G_{1}(-1)=G_{1}(1)=0, \quad G_{1}(t)= \begin{cases}1-|t|, & |t| \leqslant 1, \\ 0, & |t|>1,\end{cases}
$$

построим решение уравнения

$$
A^{m} G_{m}(t)=A\left(A^{m-1} G_{m}(t)\right)=\delta(t) .
$$

Положим $A^{m-1} G_{m}(t)=G_{1}(t)$ и через $G_{m-1}(t)$ обозначим решение уравнения $A^{m-1} G_{m-1}(t)=\delta(t)$. Тогда функцию $G_{m}(t)$ можно взять в виде свертки: $G_{m}(t)=$ $G_{m-1}(t) * G_{1}(t)$. Последовательность В-сплайнов нечетных степеней получаем с помощью рекуррентного соотношения $B_{2 m-1}(t)=G_{m}(t), G_{m}(t)=G_{m-1}(t) * G_{1}(t)$, $m=2,3, \ldots$. Непосредственньм вычислением убеждаемся, что $G_{1}(t)=B_{0}(t) * B_{0}(t)$, где

$$
B_{0}(t)=\left\{\begin{array}{cc}
1, & |t| \leqslant \frac{1}{2} \\
0, & |t|>\frac{1}{2}
\end{array}\right.
$$

- В-сплайн нулевой степени. Рекуррентная формула $B_{m}(t)=B_{m-1}(t) * B_{0}(t), m=$ $1,2, \ldots$, дает всю последовательность $\mathbf{B}$-сплайнов как нечетных, так и четных степеней. Конкретных выражений для производящих функций $\varphi(t)=B_{m}(t), m>1$, мы не выписьваем, так как они хорошо известны [5].

3) Глобально определенные составные сплайны (Н-сплайны). В качестве решения уравнения $A^{m-1} G_{m-1}(t)=\delta(t)$ возьмем $g_{2 m-3}(t)$ и тогда функцию $G_{m}(t)$ получаем в виде свертки: $G_{m}(t) \equiv H_{2 m-1}(t)=g_{2 m-3}(t) * B_{1}(t), m \geqslant 2$. Не приводя общих формул [6], ограничимся случаем $m=2$ :

$$
\varphi(t)=H_{3}(t)= \begin{cases}-\frac{|t|^{3}}{3}+t^{2}+\frac{1}{3}, & |t| \leqslant 1 \\ |t|, & |t|>1\end{cases}
$$

Сверткой $H_{2 m}(t)=g_{2 m-1}(t) * B_{0}(t)$ определим аналогичный тип сплайнов четных степеней. При $m=1$ получаем (с точностью до коэффициента)

$$
\varphi(t)=H_{2}(t)= \begin{cases}t^{2}+\frac{1}{4}, & |t| \leqslant \frac{1}{2} \\ |t|, & |t|>\frac{1}{2}\end{cases}
$$


4) Односторонние сплайны. В качестве частного решения уравнения $D G=\delta(t)$ возьмем функцию Хевисайда

$$
h(t)=\left\{\begin{array}{l}
0, t \leqslant 0 \\
1, t>0
\end{array}\right.
$$

Сплайны, построенные с помошью рекуррентных соотношений $O_{2 m}(t)=B_{2 m-1}(t) *$ $h(t)$ или $O_{2 m+1}(t)=B_{2 m-1}(t) * h(t) * h(t)$ будем назьвать односторонними. Для $m=1$

$$
\begin{aligned}
& \varphi(t)=O_{2}(t)= \begin{cases}0, & t \leqslant-1, \\
\frac{(1+t)^{2}}{2}, & -1<t \leqslant 0, \\
1-\frac{(1-t)^{2}}{2}, & 0<t \leqslant 1, \\
1, & t>1,\end{cases} \\
& \varphi(t)=O_{3}(t)= \begin{cases}0, & t \leqslant-1, \\
\frac{(1+t)^{3}}{6}, & -1<t \leqslant 0, \\
t+\frac{(1-t)^{3}}{6}, & 0<t \leqslant 1, \\
t, & t>1 .\end{cases}
\end{aligned}
$$

Односторонние сплайны удобно применять к решению задач с начальными условиями для дифференциальных уравнений (обыкновенных и с частньми производными).

Б) Гиперболические сплайны (коррелящионного типа).

5) Убьвающие на бесконечности сплайны (K-сплайны). Определяются как решения уравнения $A^{m} G_{m}(t)=\delta(t), A=-\alpha^{2} D^{2}+1$. Пусть $K_{1}(t)$ - частное решение уравнения $A K_{1}(t)=\delta(t): K_{1}(t)=1 /(2 \alpha) \cdot e^{-|t / \alpha|}$. Убывающие на бесконечности решения уравнения $A^{m} K_{m}(t)=\delta(t)$ будем строить в виде рекуррентной последовательности $K_{m+1}(t)=K_{m}(t) * K_{1}(t), m=1,2, \ldots$ В частности,

$$
\begin{aligned}
& \varphi(t)=K_{2}(t)=\frac{1}{4 \alpha} \cdot\left(1+\left|\frac{t}{\alpha}\right|\right) e^{-|t / \alpha|} \\
& \varphi(t)=K_{3}(t)=\frac{3}{16 \alpha} \cdot\left(1+\left|\frac{t}{\alpha}\right|+\frac{1}{3}\left|\frac{t}{\alpha}\right|^{2}\right) e^{-|t / \alpha|}
\end{aligned}
$$

6) Финитные сплайны (Ф-сплайны). Воспользовавшись решением краевой задачи $A \Phi_{1}(t)=\delta(t), \Phi_{1}(-1)=\Phi_{1}(1)=0$ :

$$
\Phi_{1}(t)=\frac{a}{\alpha}\left\{\begin{array}{ll}
\operatorname{sh}(1-|t / \alpha|), & |t / \alpha| \leqslant 1, \\
0, & |t / \alpha|>1,
\end{array} \quad a=\frac{1}{2(\operatorname{ch}(1)-1)},\right.
$$

финитные решения уравнения $A^{m} \Phi_{m}(t)=\delta(t)$ строим с помощью рекуррентной формулы $\Phi_{m+1}(t)=\Phi_{m}(t) * \Phi_{1}(t), m=1,2, \ldots$ В частности,

$$
\varphi(t)=\Phi_{2}(t)=\frac{a^{2}}{\alpha} \cdot\left\{\begin{array}{l}
0, \quad|t / \alpha| \geqslant 2, \\
{[(2-|t / \alpha|) \operatorname{ch}(2-|t / \alpha|)-\operatorname{sh}(2-|t / \alpha|)] / 2, \quad 1<|t / \alpha|<2,} \\
-\operatorname{ch}(t / \alpha)+\operatorname{ch}(1)[\operatorname{sh}(1-|t / \alpha|)+|t / \alpha| \operatorname{ch}(1-|t / \alpha|)] \\
-[\operatorname{sh}(|t / \alpha|)-|t / \alpha| \operatorname{ch}(t / \alpha)] / 2, \quad 0 \leqslant|t / \alpha| \leqslant 1 .
\end{array}\right.
$$


7) Сплайны с натяжением [7]. Получаются как финитные решения уравнения $A G(t)=$ $\delta(t), A=\left(-D^{2}\right)^{m-1}\left(-\alpha^{2} D^{2}+1\right)$, сверткой $N_{2 m-1}(t)=B_{2 m-3}(t) * \Phi_{1}(t), m=2,3, \ldots$ Для $m=2$ имеем

$$
\varphi(t)=N_{3}(t)=\frac{a}{\alpha}\left\{\begin{array}{l}
0, \quad|t / \alpha| \geqslant 2, \\
\operatorname{sh}(2-|t / \alpha|)-(2-|t / \alpha|), \quad 1 \leqslant|t / \alpha|<2, \\
-|t / \alpha|+2 \operatorname{ch}(1)(1-|t / \alpha|) \\
\quad-[2 \operatorname{sh}(1-|t / \alpha|)-\operatorname{sh}(|t / \alpha|)], \quad 0 \leqslant|t / \alpha|<1 .
\end{array}\right.
$$

8) Глобально определенные растущие на бесконечности сплайны. Получаем в виде решений уравнения $A G(t)=\delta(t), A=\left(-\alpha D^{2}+1\right)^{m-1}\left(-\alpha^{2} D^{2}+1\right), m=2,3 \ldots$, сверткой $S_{m}(t)=\Phi_{m-1}(t) * S_{1}(t)$ частного решения уравнения $\left(-\alpha^{2} D^{2}+1\right) S_{1}(t)=\delta(t)$, $S_{1}(t)=\operatorname{sh}(|t / \alpha|) / 2$, с $\Phi$-сплайнами. В частности, для $m=2$ получаем

$$
S_{2}(t)=\frac{a}{2 \alpha} \begin{cases}\operatorname{sh}(|t / \alpha|) \operatorname{sh}(1), & |t / \alpha| \geqslant 1, \\ {[\operatorname{ch}(1) \operatorname{ch}(t / \alpha)-|t / \alpha| \operatorname{ch}(1-|t / \alpha|)-\operatorname{sh}(|t / \alpha|),} & |t / \alpha|<1 .\end{cases}
$$

9) Сплайны переменной жесткости. Пусть $T=f(t) D^{2}, f(t) \neq 0, P=T * T=$ $\left.D^{2}\left[f^{2}(t)\right] D^{2}\right)$. Решением уравнения $P=G(t, s)=\delta(t, s)$ является функция

$$
G(t, s)=\operatorname{sgn}(t-s) \int_{s}^{t} \frac{(t-\xi)(\xi-s)}{2 f^{2}(\xi)} d \xi .
$$

В частности, положим $\left.f(t)=1+(t / \alpha)^{2}, P=T * T=D^{2}\left(1+(t / \alpha)^{2}\right)^{2} D^{2}\right)$. Поскольку коэффициенты оператора $T$ константами не являются, функция Грина $G(t, s)$ оператора $P$ не инвариантна к сдвигу относительно точки $t$ :

$$
G(t, s)=\frac{\alpha^{4}}{4}\left[\left|\frac{t}{\alpha}-\frac{s}{\alpha}\right|-\left(1+\left(\frac{t}{\alpha}\right)\left(\frac{s}{\alpha}\right)\right) \operatorname{sgn}(t-s)\left(\operatorname{arctg}\left(\frac{t}{\alpha}\right)-\operatorname{arctg}\left(\frac{s}{\alpha}\right)\right)\right] .
$$

Базисные сплайны $\varphi_{i}(t)$ образуются по правилу $\varphi_{i}(t)=G\left(t, t_{i}\right)$.

В) Многомерные сплайны.

10) Топологию пространства $\mathbf{E}$ числовых функций определим с помощью полускалярного произведения $(x \mid g)=\int_{\Omega}(\partial x, \partial g) d t$, где $\Omega$ - ограниченная область в $\mathbb{R}^{m}$, $m \geqslant 2$. Соответствуюший квадрат полунормы в $\mathbf{E}$ дается равенством

$$
N^{2}(x)=(x \mid x)=\int_{\Omega}(\partial x)^{2} d t .
$$

Тождество Грина определяет вид оператора $A=-\Delta$ :

$$
(x \mid g)=\int_{\Omega}(\partial x, \partial g) d t=-\int_{\Omega} \Delta x g d t+\int_{\partial \Omega} \frac{\partial x}{\partial \nu} g d s,
$$

где $\nu$ - нормаль к границе $\partial \Omega$. Пусть $g=0$ на $\partial \Omega$; тогда

$$
(x \mid g)=-\int_{\Omega} \Delta x g d t=(-\Delta x, g) .
$$


В рассмотренной постановке задачи $X$ - это плотноев $\mathbf{E}$ (отделимое) подпространство функций, принимающих нулевые значения на границе области $\Omega$, оператор $A: X \rightarrow$ $L^{2}(\Omega)$ - непрерьвный оператор $-\Delta$, действующий из $X$ в $L^{2}(\Omega)$ и симметрический на элементах $X:(-\Delta x, g)=(x,-\Delta g), x, g \in X,\|x\|=N(x), x \in X$ - норма на $X$. Пусть $\mathbf{M}$ - подпространство функций из $L^{2}(\Omega)$, принимающих нулевые значения на гранище $\partial \Omega$. Поскольку справедливы вложения $\mathbf{D}(\Omega) \subset X \subset \mathbf{M} \subset L^{2}(\Omega)$ и $\mathbf{D}(\Omega)$ (пространство основных функций) всюду плотно в $L^{2}(\Omega)$, то $X$ всюду плотно в $\mathbf{M}$ и $L^{2}(\Omega)$. Рассмотрим непрерывное расширение линейной формы $g \rightarrow(x,-\Delta g)$ с $X$ на $\mathbf{M}$ с сохранением равенства $(-\Delta x, g)=\langle x,-\Delta g\rangle, x \in X, g \in \mathbf{M}$. Тем самым определен сопряженньй оператор $A^{\prime}$, имеющий ту же самую дифференциальную форму, что и $A$ и действующий из $\mathbf{M}$ в $\mathbf{E}^{\prime}$. Если $\delta_{t} \in A^{\prime}(\mathbf{M}) \subset \mathbf{E}^{\prime}$, то уравнение $A^{\prime} G_{t}=\delta_{t}, G_{t} \in \mathbf{M}$, разрешимо и равенство $x=\left(-\Delta x, G_{t}\right)=\left(h, G_{t}\right)$ определяет оператор $G$, действующий из $A(X) \subset L^{2}(\Omega)$ на $X$.

В силу плотного вложения $X$ в $\mathbf{E}$ и $\mathbf{M}$ в $L^{2}(\Omega)$ равенство $(\Delta x, g)=\langle x,-\Delta g\rangle, x \in X$, $g \in \mathbf{M}$, сохраняется, если в нем граничные условия исключаются из рассмотрения, и тогда $x=\left(h, G_{t}\right), x \in \mathbf{E}, G_{t} \in L^{2}(\Omega)$, где $h \in A(\mathbf{E}) \subset L^{2}(\Omega)$ и $G_{t}$ - некоторое частное решение уравнения $A^{\prime} G_{t}=\delta_{t}$. При $\delta_{t} \in A^{\prime}\left(L^{2}(\Omega)\right) \subset \mathbf{E}^{\prime}$ его решения существуют. Возьмем частное решение $g_{t}$ этого уравнения, когда ядро оператора $A^{\prime}$ полагается равным нулю. Оно имеет вид $g(t, s)=\ln (t-s) /(2 \pi)$ при $m=2$ и $g(t, s)=-1 /(\mid t-$ $\left.\left.s\right|^{m-2}(m-2) \sigma_{m}\right), m>2$, где $\sigma_{m}-$ площадь поверхности единичной сферы в $\mathbb{R}^{m}$. Функция $g_{t}$ сингулярна в точке $s=t$ и поэтому пространству $\mathbf{E}$ не принадлежит. Очевидно, что она является элементом пространства $L^{2}(\Omega)$.

Пусть информация о искомой функции $x(t)$ нам известна в виде значений интегралов

$$
f_{i}(x)=\left(h_{i}, x\right)=\int_{\Omega} h_{i}(t) x(t) d t=\alpha_{i}, \quad i=1, \ldots, n, \quad \Omega \subset \mathbb{R}^{m}
$$

Решение $x(t)$ этой задачи строим в виде разложения $x(t)=\sum_{i=1}^{n} \lambda_{i} \varphi_{i}(t), \varphi_{t}(t)=f_{i}\left(g_{t}\right)$, $i=1, \ldots, n, t \in \Omega$. Коэффициенты $\lambda=\left(\lambda_{i}\right)$ находим из условий $(20)$.

Приведем численную реализацию описанного алгоритма. Будем считать для простоты, что $\Omega-m$-мерньй куб. Рассмотрим разбиение области $\Omega$ сеткой с равномерным и одинаковьм по всем координатам шагом $\tau$. Каждую построенную таким образом подобласть будем нумеровать индексом $i$, а через $t_{i}$ будем обозначать точку центра $i$-й подобласти. Обозначим через $r_{i}=\left|t-t_{i}\right|$ расстояние между точками $t$ и $t_{i}$ в евклидовой метрике и возьмем линейно независимую систему функций

$$
h_{i}(t)=\left\{\begin{array}{c}
1, r_{i} \leqslant \frac{\tau}{2}, \\
0, r_{i}>\frac{\tau}{2},
\end{array} \quad i=1, \ldots, n, \quad t \in \mathbb{R}^{m}\right.
$$

где $n$ - число подобластей разбиения области $\Omega$.

Базисные функции $\varphi_{i}(t)$ для двух случаев $m=2, m>2$ выгислим непосредственно из уравнения $-\Delta \varphi(t)=h(t)$, где $h(t)=h_{0}(t)$ и $\tau$ взято равным единице. В силу симметрии задачи оно приводится к обькновенному дифференциальному уравнению относительно функции $\varphi(r)$, частное решение которого (производящую функцию) выберем из 
условий $\varphi(0)=\varphi^{\prime}(0)=0$. Преобразования сдвига и масштабирования производяшей функции $\varphi(r)$ приводят к следующим выражениям для базисных функций:

$$
\begin{aligned}
& \varphi_{i}(t)=\frac{1}{2}\left\{\begin{array}{ll}
\left(\left|t-t_{i}\right| / \varepsilon\right)^{2}, & \left|t-t_{i}\right| \leqslant \varepsilon, \quad \varepsilon=\frac{\tau}{2}, \\
\ln \left(\left|t-t_{i}\right| / \varepsilon\right)^{2}+1, & \left|t-t_{i}\right|>\varepsilon,
\end{array} \quad t \in \mathbb{R}^{2},\right. \\
& \varphi_{i}(t)=\frac{1}{2}\left\{\begin{array}{ll}
\left(\left|t-t_{i}\right| / \varepsilon\right)^{2}, & \left|t-t_{i}\right| \leqslant \varepsilon, \\
\frac{1}{m-2}\left[m-2\left(\varepsilon /\left|t-t_{i}\right|\right)^{m-2}\right], & \left|t-t_{i}\right|>\varepsilon,
\end{array} \quad t \in \mathbb{R}^{m}, \quad m>2 .\right.
\end{aligned}
$$

Они линейно независимы, непрерывно дифференцируемы и построенная по ним линейная оболочка порождает в $\mathbf{E}$ алгебраические сплайны класса $C^{1}(\Omega)$.

\section{СПИСОК ЦИТИРОВАННОЙ ЛИТЕРАТУРЫ}

[1] Дэй М. М. Нормированные линейные пространства. М.: ИЛ, 1961.

[2] Колесников А. П. Алгебраические базисы в векторных пространствах // Вестн. РУДН. 1997. Т. 97. №1. С. 78-86.

[3] Колесников А. П. Функциональные сплайны. М.: Изд. РУДН, 1993.

[4] Эдвардс Р. Функциональный анализ. Теория и приложения. М.: Мир, 1969.

[5] Марчук Г.И., Агошков В.А. Введение в проекционно сеточные методы. М.: Наука, 1981.

[6] Колесников А. П. Топологические методы в теории приближений и численном анализе. М.: Изд. УРСС, 2001.

[7] Schweikert D. J. An interpolation curve using a spline in tension // J. Math. Phys. 1966. V. 45 . P. $312-317$.

Российский университет дружбы народов

Поступило

E-mail: akolesnikov@sci.pfu.edu.ru

28.01.1999

Исправленный вариант

21.03.2002 